\title{
Exploring the chemo-textural familiarity hypothesis for scorpion navigation
}

\author{
Douglas D. Gaffin and Brad P. Brayfield: Department of Biology, University of Oklahoma, Norman, OK 73019 USA;
}

E-mail: ddgaffin@ou.edu

\begin{abstract}
The navigation by scene familiarity hypothesis provides broad explanatory power for how bees and ants navigate from the hive to distant food sources and back. The premise is that the visual world is decomposed into pixelated matrices of information that are stored and readdressed as the insects retrace learned routes. Innate behaviors in these insects (including learning walks/flights and path integration) provide the important goal-directed views to allow the initial retracing (i.e., the insect must learn the scene while moving toward the goal because everything looks different while moving away). Scorpion navigation may use a similar premise, with the chemical and textural features of the environment substituting for visual input. Scorpion pectines support thousands of chemo- and mechano-sensitive units called peg sensilla, each containing at least 10 energetically expensive sensory neurons. We have long wondered why pectines have so many pegs and associated neurons. Many sand scorpions emerge onto the surface from their home burrows at night to pursue insect prey and somehow find their way back to their burrows. Based on the measured resolution of peg sensilla, we have calculated that sufficient information exists in sand's texture to enable scorpions to retrace previously experienced paths with little to no chance of confusion. Preliminary evidence of learning walks and path integration in scorpions is also congruent with the navigation by chemo-textural familiarity hypothesis.
\end{abstract}

Keywords: Behavior, electrophysiology, homing, sensory, pectines

Scorpion pectines are ornate, ground-facing chemosensory appendages (Cloudsley-Thompson 1955) that support tens of thousands of chemo-tactile sensilla called "pegs" (Foelix \& Müller-Vorholt 1983; Gaffin \& Brownell 1997, 2001; Wolf 2008). A lingering question is: Why are there so many pegs? Here, we offer a novel chemo-textural familiarity hypothesis for pecten function. We propose that scorpions may use their pectines to acquire and process ground-based chemical and textural information and use it to recapitulate learned routes to their home burrows.

We derived this idea from the Navigation by Scene Familiarity Hypothesis (NSFH) proposed for homing insects (Baddeley et al. 2012). The premise is that a foraging animal, such as a bee or an ant, uses its compound eyes to acquire matrices of visual information while moving toward a goal. Then, during retracing runs, the animal moves in a direction that minimizes the pixel-by-pixel difference between its current retinal view and the views it previously stored in memory. This simple behavioral rule is elegant and congruent with the limited neural capacity of an insect's brain. The animal is not burdened with memorizing long sequences of scenes. Rather, it exploits the inherent stability and visual richness of natural scenes to help it retrace its original path (Gaffin et al. 2015).

Similarly, we suggest that scorpions might use their pectines to acquire and store matrices of chemical and textural information during homebound journeys and use these memories to retrace paths by moving in a direction that minimizes differences between current pecten "glimpses" and those in memory. However, for this mechanism to be viable, three prerequisites should be met. First, the scorpion needs sufficient sensory receptors to detect and encode appropriate environmental complexity. Second, the environment needs to be sufficiently complex to not confuse the animal in areas with similar patterns. And finally, the animal must have a way to acquire goal-directed information, such as through innate behaviors like path integration (Müller \& Wehner 1988) and/ or learning walks (Wehner et al. 2004). Here we draw parallels between what is known for insect navigation by visual familiarity and mounting evidence suggesting that scorpions might navigate by chemo-textural familiarity.

Sufficient sensory receptors. - For the NSFH to be plausible, sensor resolution must suit the complexity of the animal's environment. In insects, dense packing of light-sensing units called ommatidia enable high resolution. Each ommatidium is directed at its own blurred piece of the visual world; together, ommatidia give the compound eyes a semi-panoramic view of the world (Seidl \& Kaiser 1981). The number of ommatidia per eye is impressive. Desert ants have about $\sim 500$ (Schwarz et al. 2011), houseflies 3500 (Sukontason et al. 2008), honeybees $\sim 5,500$ (Seidl \& Kaiser 1981), and dragonflies 24,000 ommatidia in each eye (Pritchard 1966)! Consider a desert ant and the 1000 ommatidia that combine to compose her two eyes. If each ommatidium is configured to respond in only two states - "off" if its face is mostly dark and "on" if mostly light - then the number of uniquely detectable patterns is $2^{1000}$. Of course, this number of patterns would multiply significantly if the number of detectable states extends to multiple gray levels and/or to colors.

A legitimate question would be how this pattern-detection potential compares to the number of scenes an insect experiences. Worker ants forage for a couple of months. If we assume an individual forages full time (day and night), and her eyes capture frames at 60 per second (Srinivasan \& Lehrer 1984), she would experience about 300 million glimpses in her lifetime $(24 \mathrm{~h} /$ day $\times 160 \mathrm{~min} / \mathrm{h} \times 60 \mathrm{~s} / \mathrm{min} \times 60$ glimpses/s). Simply put, the pattern detection potential of an ant's eyes is hundreds of orders of magnitude greater than the number of scenes she experiences.

Scorpion pectines also possess a high degree of sensor resolution. The pectines are divided into a species-dependent number of teeth, ranging from a half dozen in some members of the Chactidae (Swoveland 1978) to nearly 40 in some male Vaejovidae (Gaffin \& Brownell 2001). Each tooth supports an array of minute, chemo-tactile sensitive peg sensilla on its 

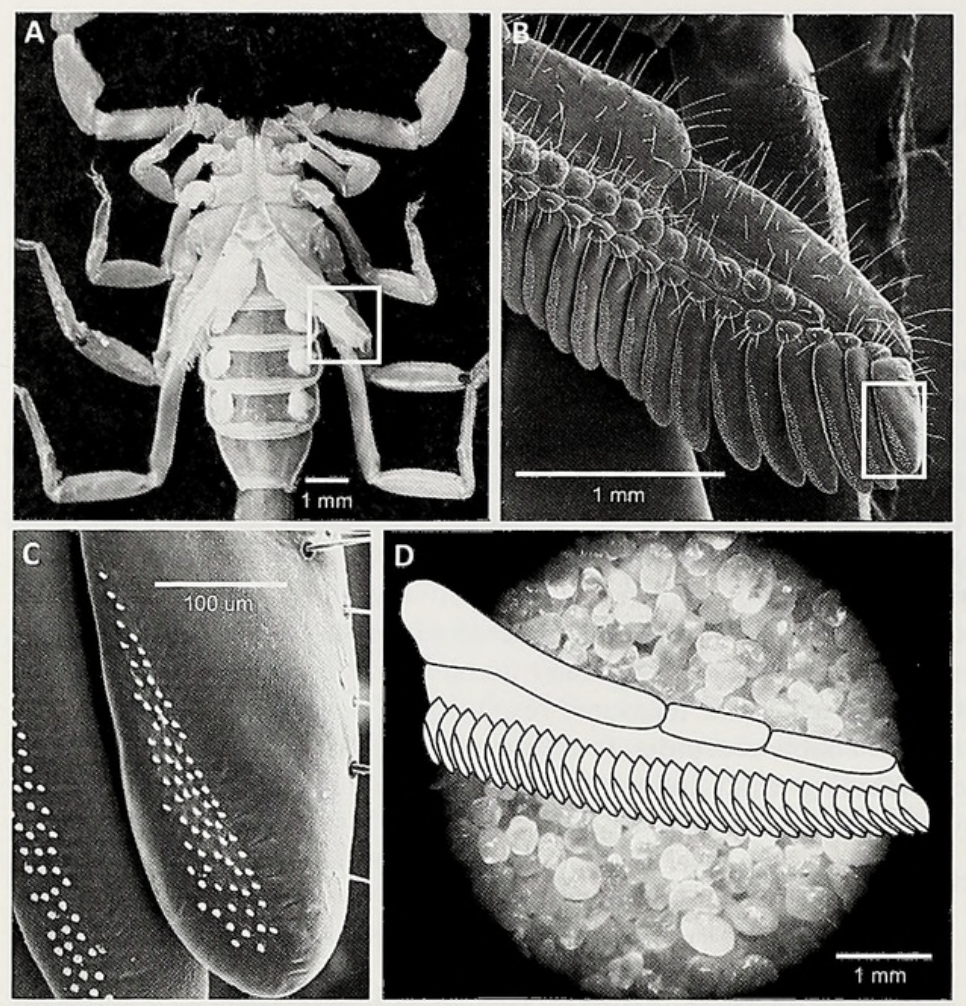

Figure 1.-Scorpion pectines have sufficient sensory receptors. (A) Ventral view of a male $P$. utahensis shows the paired pectines extending laterally from the ventral mesosoma. (B) Expanded view from (A); each pecten is composed of a series of teeth. (C) Expanded view from (B); each tooth has dense patches of minute peg sensilla. (D) Size of a male $P$. utahensis pecten relative to sand grains from the animal's native west Texas habitat. [1(B) and 1(C) provided by Elise Knowlton.]

distal, ground-directed surface (Fig. 1). Like the ommatidia of insect eyes, the number of peg sensilla on pectinal teeth is impressive. The number of pegs varies by species from tens to hundreds per tooth and from hundreds to tens of thousands across the two pectines (Gaffin \& Brownell 2001). We have estimated that female Paruroctonus utahensis (Williams, 1968) have approximately $12,500 \mathrm{pegs} / \mathrm{mm}^{2}$, which translates to tens to hundreds of pegs per grain of sand in the perceived environment (Gaffin \& Walvoord 2004).

The pegs are richly innervated by populations of sensory neurons. Each peg contains at least one mechanosensitive neuron that responds to peg deflection (Hoffmann 1964; Foelix \& Müller-Vorholt 1983; Gaffin \& Brownell 1997; Gaffin 2002; Melville 2000). Most peg neurons (10 or more) have typical chemosensory characteristics based on morphological accounts (Foelix \& Müller-Vorholt 1983). Judged by their single, slit-shape terminal pore, peg sensilla are best classified as contact chemoreceptors. Peg neurons show broad response profiles to near-range stimulation by a variety of volatile organic compounds (Gaffin \& Brownell 1997) or by direct contact of chemicals with the peg tip (Knowlton \& Gaffin 2011). In short, scorpion pectines have the neural potential to detect enormous numbers of unique chemotextural surface patterns.

If we are to understand how information is relayed and processed in the scorpion brain, it will be useful to develop electrophysiological techniques to record along specific parts
Table 1.-Potential number of unique response patterns of scorpion pectines, given various numbers of teeth and neural response states $(n=$ no. of teeth).

\begin{tabular}{ccccc}
\hline & & \multicolumn{3}{c}{$\begin{array}{c}\text { Number of unique patterns } \\
\text { at } 2,10, \& 100 \text { response states }\end{array}$} \\
\cline { 3 - 5 } $\begin{array}{c}\text { \# of teeth } / \\
\text { pecten }\end{array}$ & $\begin{array}{c}\text { \# of teeth } / \\
2 \text { pectines }\end{array}$ & $\begin{array}{c}2 \text { states } \\
\left(2^{n}\right)\end{array}$ & $\begin{array}{c}10 \text { states } \\
\left(10^{n}\right)\end{array}$ & $\begin{array}{c}100 \text { states } \\
\left(100^{n}\right)\end{array}$ \\
\hline 20 & 40 & $\sim 10^{12}$ & $10^{40}$ & $10^{80}$ \\
25 & 50 & $\sim 10^{15}$ & $10^{50}$ & $10^{100}$ \\
30 & 60 & $\sim 10^{18}$ & $10^{60}$ & $10^{120}$ \\
35 & 70 & $\sim 10^{21}$ & $10^{70}$ & $10^{140}$ \\
40 & 80 & $\sim 10^{24}$ & $10^{80}$ & $10^{160}$ \\
\hline
\end{tabular}

of the pectinal neural pathway. Currently, we hypothesize that the unit of information is the individual pectinal tooth rather than the individual peg. Morphological tracings of pectinal neurons to the scorpion's subesophageal ganglion show that a topological arrangement is maintained at that level (Brownell 1998). That is, the order of the teeth on each pecten spine appears to be preserved in the brain, where the pectinal nerve terminates prior to subsequent secondary neural processing. Enormous amounts of information are still available, even if peg responses are averaged across the breadth of each tooth, and such blurring of resolution could help compensate for environmental disturbance. Table 1 shows sample combinations of pecten tooth configurations and different assumptions for neural response states $(2,10$, and 100 states $)$ in the brain. For example, an animal with only 20 teeth per pecten (i.e., 40 teeth across both pectines) and a simple all-or-none (2-state) response rule, can detect $10^{12}$ different patterns. If the number of response states increases to 10 , then the number of resolvable patterns grows to $10^{40}$. Eighty pectinal teeth and 100 response states could accommodate $10^{160}$ different patterns!

Sufficient environmental complexity.-For accurate navigation by scene familiarity to be plausible, the environment must contain enough information that the animal will not get lost or confused by moving toward a similar-looking (or similartasting) but incorrect scene. When natural scenes (be they visual, chemical, or textural) are transformed to pixelated matrices, the chance that any two scenes will be alike must be extremely remote.

When considered from the pixelated matrices of an insect's eye, the world is rich in visual information. This content can be assessed by transforming scenes to matrices of various dimensions and calculating the summed absolute pixel-bypixel differences among the scenes (Zeil et al. 2003; Baddeley et al. 2012; Narendra et al. 2013; Gaffin et al. 2015; Gaffin \& Brayfield 2016). An individual scene compared to all other scenes produces a volcano-shaped plot. The focal scene forms the volcano's pit (subtracting a matrix of pixels from itself yields zero) and the summed difference values that vary directly with distance, form the caldera's slopes (Zeil et al. 2003; Narendra et al. 2013). Extending this type of analysis to a path of images produces a canyon-shaped plot, where the river bottom reflects the self-subtraction of the path scenes and the slopes reflect the increasing differences with distance from the path. Using this information, autonomous agents can be 

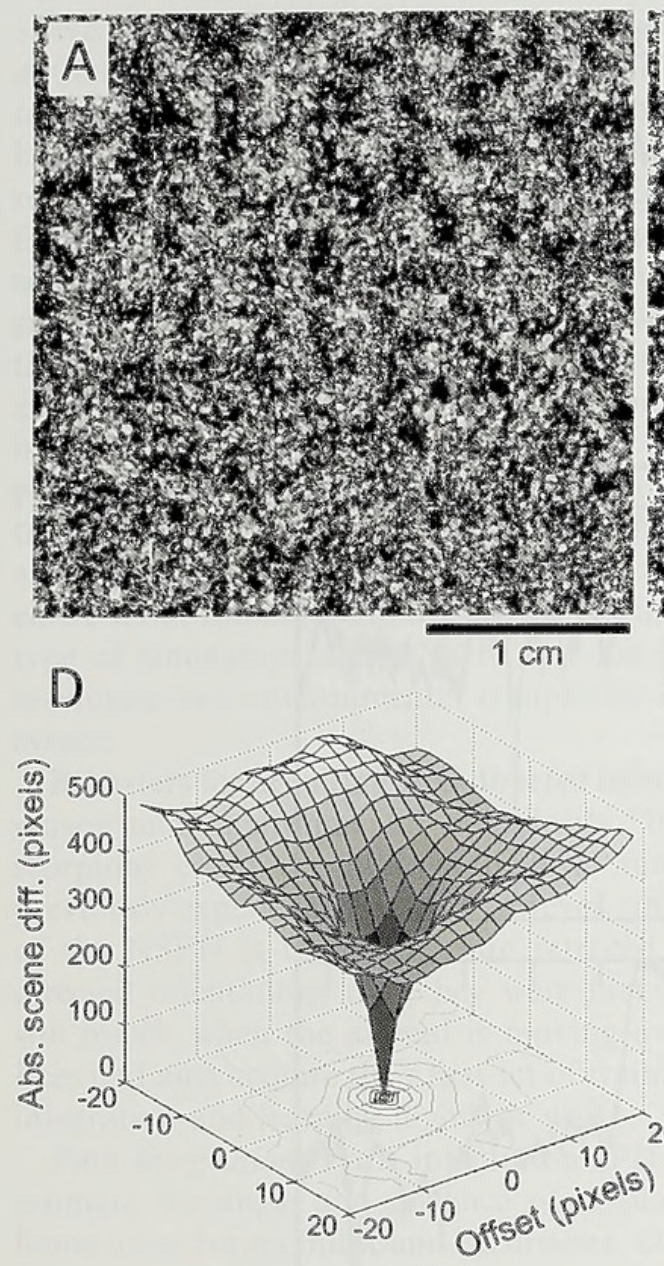

Figure 2.-Analysis of textural information content. (A) High contrast photography was used to estimate sand textural information. Sand from Monahans State Park (TX) was placed in a Petri dish and illuminated by light directed from the side at $30^{\circ}$ to the sand surface. We took 100 random photographs of the sand using a $40 \mathrm{x}$ power dissecting scope. We used a MATLAB script to import, turn to gray, crop to squares representing $3 \times 3 \mathrm{~mm}$ of sand surface, enhance the contrast, and reduce the resolution to $335 \times 335$ pixels for each of the 100 images. This resolution value was derived by multiplying the peg sensilla density of 12,500 per $\mathrm{mm}^{2}$ (Gaffin \& Walvoord 2004 ) by $9 \mathrm{~mm}^{2}$ and taking the square root. We randomly rotated and concatenated the squares to produce a 10x10 matrix of these images composed of $11,222,500$ pixels in a $3350 \times 3350$ square. Using a value of eight pegs for chemical discrimination (Knowiton \& Gaffin 2011), the number of pixels was reduced to $1,402,813$ in the square $(11,222,500 / 8)$. The square root of this number produces a matrix of 1184 pixels on each side. (B) We changed the image to black and white and superimposed a drawing of the pectines to show relative scale. The two rectangles indicate the area of the peg fields, and these have been combined into the square below (area $\sim 1.5 \mathrm{~mm}^{2}$ ). (C) Expansion of area around the square from (B). This square is 30 pixels on a side, which represents our sensor resolution based on the resolving power of peg sensilla. (D) Image difference volcano indicates that the focal scene is different from all other scenes in a 1600-pixel area. Surface (E) and contour (F) plots of difference information for a 200-pixel-long diagonal training path across a $400 \times 400$ (160,000 pixels) piece of the sand landscape. programed to use these catchment areas to navigate based on scene familiarity alone (Baddeley et al. 2012; Gaffin et al. 2015; Gaffin \& Brayfield 2016).

What about the chemo-textural landscape that scorpion pectines encounter? While the exact composition, concentration, and placement of naturally occurring chemicals on sand is beyond our reach, we can estimate textural information using high contrast photography. To do this, we placed sand from the scorpion's habitat in a Petri dish, directed a light from the side, and took 100 random photographs of the sand under a $40 \mathrm{x}$ scope. We then wrote a MATLAB script to import, crop, turn to gray, enhance the contrast, randomly rotate, and stitch together the photos to create a proxy for the sand's textural landscape (Fig. 2A). We then reduced the resolution of this landscape based on the measured chemicalresolving power of the pegs. We have estimated that it requires at least eight pegs to discern two chemicals based on the response of individual pegs to pure stimuli and the time that the pegs are close enough to the ground during a "pectinal sniff" to register a response (Knowlton \& Gaffin 2011). To further simplify the landscape and to be as conservative as possible for this simulation, we converted the image to black and white (shown in Fig. 2B). This modified image represents the details of the textural world from the pectines' point of view. We then calculated the area of the ground-contacting surfaces of pectinal teeth and generated a square of the same area (see the small square below the superimposed pectines in Fig. 2B). Figure 2C shows an expanded sample of the textural landscape relative to this square to highlight what the patterns look like at that level.

By comparing individual pecten-sized squares in the sand landscape to all other possible squares, we generated 

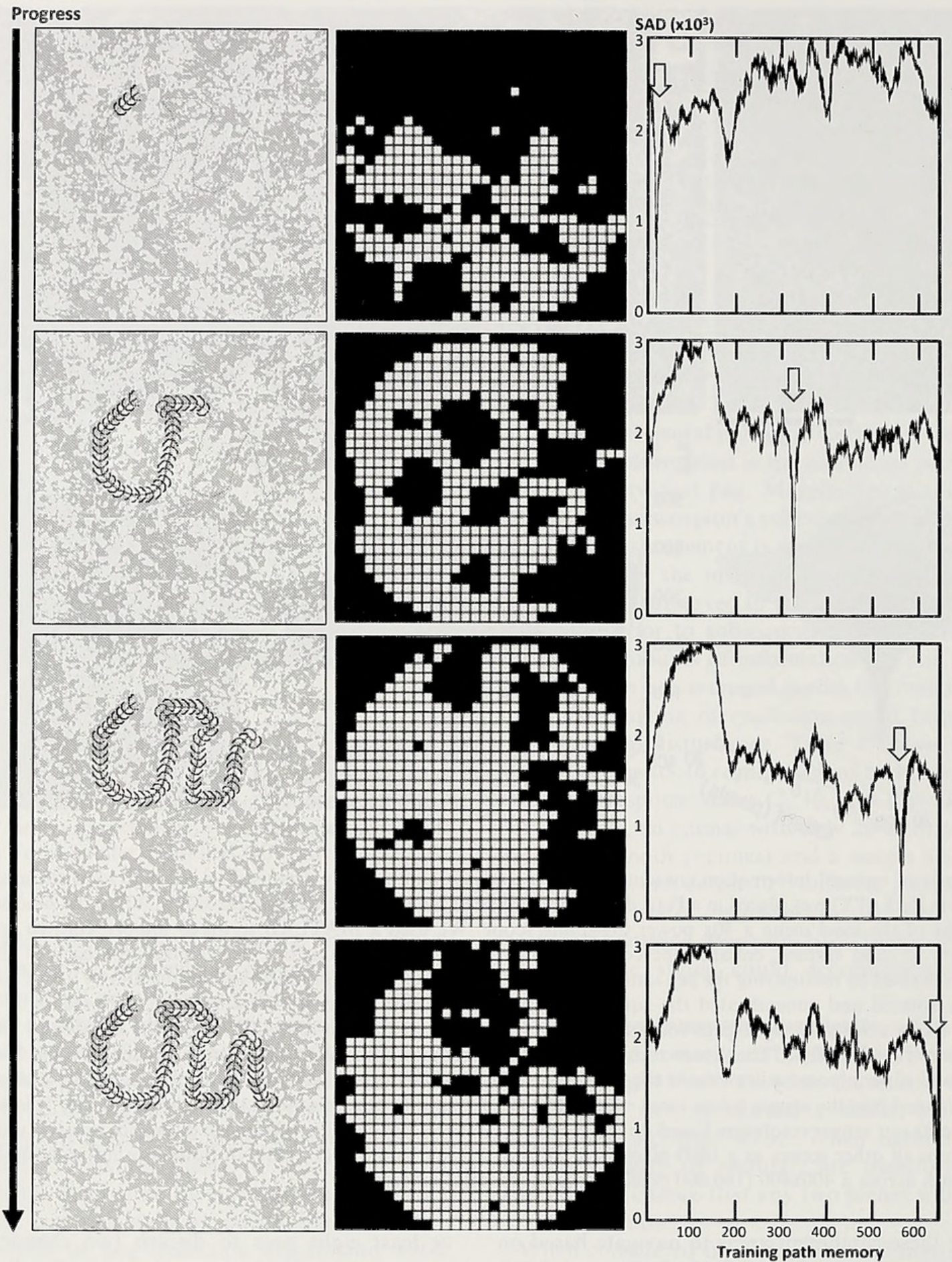

Figure 3.-Auto-tracking of a sample training path. A complex training path was drawn over the sand textural landscape (from Fig. 2(B)) to initiate the simulation. In this example, approximately 700 pecten-sized "scenes" were pixelated (30x30), circularized and stored to memory. The circularization simplifies rotational comparisons of samples. The agent is placed near the beginning of the training path and an arc of samples is taken to mimic an animal's saccade. Each test scene matrix is rotated and compared (non-sequentially) to all scene matrices in memory based on the sum of the absolute pixel differences (SAD). The agent selects and uses the rotational angle of the best matched test scene as its bearing for its next forward step (based on a pre-defined length). It takes a step and the saccade and testing procedure repeats. The four horizontal panels of this simulation show the progress of the agent as it successfully navigates the training path. Within each horizontal panel, the current progress of the agent (left image), the current pecten-sized pixelated "view" of the sensor (middle image), and an activity monitor that plots the SAD of the current scene compared to all scenes in memory (right image) are displayed. Note how the trough (lowest SAD) in the plot (indicated by arrow) moves in register with the progress of the agent along the training path.

a plot that resembles a volcano, similar to the one described for visual information. This plot demonstrates that each pecten-sized square is distinct from all other squares (Fig. 2D). Further, when we compared a contig- uous line of these squares (analogous to a training path) to all other squares, the plot resembled a steep-walled canyon (Fig. 2E, F) with a distinct catchment area relative to the referenced squares. 
We have extended this analysis to produce simulations of autonomous agents that retrace paths drawn over our sand texture landscape. Figure 3 shows an example of a familiaritybased tracking algorithm (using vision as a proxy for texture) retracing a complex path (adapted from Gaffin et al. 2015; see figure legend for details of how the algorithm works). The simulation shows that the inherent information content of our simplified sand texture landscape is sufficient for an analog of the pectines to navigate the training path. Of note, see the activity plots on the right of each of the four panels. The arrow in each plot highlights the trough in the sum of the absolute pixel differences (SAD) of each test scene as compared to all training scenes in memory. This again shows the catchment area that exists around each considered point relative to the entire set of training path images (as discussed in Fig. 2). This type of simulation allows us to test the interplay of sensor resolution and environmental complexity on tracking performance.

Behaviors for acquiring goal-directed information.-Based on sensor and environmental complexity, it is plausible that scorpions could use at least textural familiarity to retrace previously experienced paths. However, an important premise of the NSFH is that scenes are retraced in the same goaldirected orientations that they were first acquired; no scene will match when the animal is moving away from the goal. Bees and ants acquire their first set of training images via path integration and learning flights or walks.

Path integration: Path integration (PI) allows animals to estimate the angle and distance of displacement from their home nests during outbound excursions. Displacement studies have been used to assess PI. In a classic example, researchers caught a desert ant that stumbled on a feeder and then displaced the ant to a distant release point (Müller \& Wehner 1988). The ant walked in a straight line before making tight turning movements; the direction and length of the straight segment was approximately the same as the original direction and distance to the nest from the feeder. Desert ants appear to retrace familiar visual paths learned during training excursions that were produced initially by PI (Collett \& Collett 2000; Wehner \& Srinivasan 2003; Wittlinger et al. 2006; Baddeley et al. 2012). Consistent with the familiarity hypothesis, PI allows the individual to acquire a goal-directed set of images for subsequent retracing.

We have seen some examples of apparent PI in scorpions in the field. In these cases, we dragged a small stick across the sand to entice a scorpion from its burrow and onto a small cutting board buried just under the sand. We displaced the scorpion by carefully sliding the board $20-50 \mathrm{~cm}$ further from its burrow. In each case, the initial movement of the animal was to a point where the burrow would have been had the animal not been displaced. This straight segment was followed by looping patterns of movement. The tight turns and loops are similar to those observed in nest-searching ants (Wehner \& Srinivasan 1981).

Learning walks/flights: Bees do an interesting set of orientation flights during their first excursions from the hive (Zeil et al. 1996; Palikij et al. 2012). They back away from the hive and make short swooping arcs while facing the hive. They then return to the hive and make additional, wider sets of arcs before setting off on their first foraging trip. It is thought that they are widening the visual target for their hive, broadening the visual catchment area of familiarity that will lead them home. Similarly, desert ants do learning walks around their nests prior to setting out on their initial foraging ventures (Wehner et al. 2004).

We have, on occasion, observed what appear to be learning walks by scorpions during our scorpion hunts. Sometimes, during early evening, we have noticed animals making short looping movements away from their burrows and back. We have also captured several examples of this behavior during long-term video recordings in the lab. The movements range from tight backing movements near the burrow to looping movements that take the animal approximately $15 \mathrm{~cm}$ from the burrow before returning. If we are to fully characterize the use of PI and learning walks in scorpions, we need to document many additional examples of these behaviors in the field. It is also crucial to develop carefully controlled behavioral assays in the lab (Vinnedge \& Gaffin 2015) to precisely map PI and learning walks and to explore the use of previously experienced chemo-textural patterns during homebound journeys.

Conclusions. - In summary, several tantalizing clues allow us to think about the pectines in relation to chemo-textural familiarity navigation. Pectines are replete with thousands of chemo-tactile peg sensilla, and a conservative estimate of environmental texture indicates abundant information content based on measured sensilla resolution. There are also hints of innate path integration and learning walk behaviors that generate initial sets of goal-directed images. Still, more research is necessary to test this hypothesis properly. We need carefully controlled behavioral studies examining the use of previously experienced chemo-textural patterns during homing runs and an assay that teases apart the contributions of PI, familiarity, and other potential mechanisms (vision, chemical trails) for homing.

\section{ACKNOWLEDGMENTS}

We thank Mariëlle Hoefnagels for her valuable review of this manuscript. We also thank Andrew Philippides, Paul Graham, Alex Dewar, and Arthur Gaffin for important discussions on visual familiarity, Bob Suter for informal conversations about these ideas and for technical assistance, and Kendall Hughes for additional feedback on the narrative. Finally, we thank the Life Fund of the University of Oklahoma Foundation and professional funds from the University of Oklahoma Presidential Teaching Fellowship in the Honors College for financial support.

\section{LITERATURE CITED}

Baddeley, B., P. Graham, P. Husbands \& A. Phillippides. 2012. A model of ant route navigation driven by scene familiarity. PLoS Computational Biology 8:1-16 e1002336.

Brownell, P.H. 1998. Glomerular cytoarchitectures in chemosensory systems of arachnids. Annals of the New York Academy of Science 855:502-507.

Cloudsley-Thompson, J.L. 1955. On the function of the pectines of scorpions. Annals \& Magazine of Natural History 8:556-560.

Collett, T.S. \& M. Collett. 2000. Path integration in insects. Current Opinion in Neurobiology 10:757-762.

Foelix, R.F. \& G. Müller-Vorholt. 1983. The fine structure of 
scorpion sensory organs. II. Pecten sensilla. Bulletin of the British Arachnological Society 6:68-74.

Gaffin, D.D. 2002. Electrophysiological analysis of synaptic interactions within peg sensilla of scorpion pectines. Microscopy Research and Technique 58:325-334.

Gaffin, D.D. \& B.P. Brayfield. 2016. Autonomous visual navigation of an indoor environment using a parsimonious, insect inspired familiarity algorithm. PLoS ONE 11(4): e0153706. doi:10.1371/ journal.pone. 0153706

Gaffin, D.D. \& P.H. Brownell. 1997. Response properties of chemosensory peg sensilla on the pectines of scorpions. Journal of Comparative Physiology A 181:291-300.

Gaffin, D.D. \& P.H. Brownell. 2001. Chemosensory behavior and physiology. Pp. 184-203. In Scorpion Biology and Research. (P.H. Brownell \& G.A. Polis, eds.). Oxford University Press, New York, New York.

Gaffin, D.D. \& M.E. Walvoord. 2004. Scorpion peg sensilla: are they the same or are they different? Euscorpius 17:7-15.

Gaffin, D.D., A. Dewar, P. Graham \& A. Philippides. 2015. Insectinspired navigation algorithm for an aerial agent using satellite imagery. PLoS ONE 10(4): e0122077, doi:10.1371/journal.pone. 0122077

Hoffmann, C. 1964. Zur funktion der kammformigen organ von skorpionen. Naturwissenschaften 7:172.

Knowlton, E.D. \& D.D. Gaffin. 2011. Functionally redundant peg sensilla on the scorpion pecten. Journal of Comparative Physiology A 197:895-902.

Melville, J. 2000. The pectines of scorpions: Analysis of structure and function. Ph.D. Thesis, Oregon State University, Corvallis, OR.

Müller, M. \& R. Wehner. 1988. Path integration in desert ants, Cataglyphis fortis. Proceedings of the National Academy of Science 85:5287-5290.

Narendra, A., S. Gourmaud \& J. Zeil. 2013. Mapping the navigational knowledge of individually foraging ants, Myrmecia croslandi. Proceedings of the Royal Society B 280:20130683.

Palikij, J., E. Ebert, M. Preston, A. McBride \& R. Jander. 2012. Evidence for the honeybee's place knowledge in the vicinity of the hive. Journal of Insect Physiology 58:1289-1298.

Pritchard, G. 1966. On the morphology of the compound eyes of dragonflies (Odonata: Anisoptera), with special reference to their role in prey capture. Proceedings of the Royal Entomological Society of London A 41:1-8.
Schwarz, S., A. Narendra, A. \& J. Zeil. 2011. The properties of the visual system in the Australian desert ant Melophorus bagoti. Arthropod Structure \& Development 40:128-134.

Seidl, R. \& W. Kaiser. 1981 Visual field size, binocular domain and the ommatidial array of the compound eyes in worker honey bees. Journal of Comparative Physiology A 143:17-26.

Srinivasan, M.V. \& M. Lehrer. 1984. Temporal acuity of honeybee vision: behavioural studies using moving stimuli. Journal of Comparative Physiology A 155:297-312.

Sukontason, K.L., T. Chaiwong, S. Piangjai, S. Upakut, K. Moophayak \& K. Sukontason. 2008. Ommatidia of blow fly, house fly, and flesh fly: implication of their vision efficiency. Parasitology Research 103:123-131.

Swoveland, M.C. 1978. External morphology of scorpion pectines. Master's thesis, California State University, San Francisco.

Vinnedge, J. \& D.D. Gaffin. 2015. Determination of in-lab site fidelity and movement patterns of Paruroctonus utahensis. Journal of Arachnology 43:54 58.

Wehner, R. \& M.V. Srinivasan. 1981. Searching behaviour of desert ants, genus Cataglyphis (Formicidae, Hymenoptera). Journal of Comparative Physiology 142:315-338.

Wehner, R. \& M.V. Srinivasan. 2003. Path integration in insects. Pp. 9-30. In The Neurobiology of Spatial Behaviour. (K.J. Jeffery, ed.). Oxford University Press, New York, New York.

Wehner, R., C. Meier \& C. Zollikofer. 2004. The ontogeny of foraging behavior in desert ants, Cataglyphis bicolor. Ecological Entomology 29:240-250.

Wittlinger, M., R. Wehner \& H. Wolf. 2006. The ant odometer: stepping on stilts and stumps. Science 312:1965-1967.

Wolf, H. 2008. The pectine organs of the scorpion, Vaejovis spinigerus: structure and (glomerular) central projections. Arthropod Structure \& Development 37:67-80.

Zeil, J., M.I. Hofmann \& J.S. Chahl. 2003. Catchment areas of panoramic snapshots in outdoor scenes. Journal of the Optical Society of America A 20:450 469.

Zeil, J., A. Kelber \& R. Voss. 1996. Structure and function of learning flights in bees and wasps. Journal of Experimental Biology 199:245-252.

Manuscript received 30 September 2016, revised 19 May 2017. 


\section{$2 \mathrm{BHL}$ Biodiversity Heritage Library}

Gaffin, Douglas D and Brayfield, Brad P. 2017. "Exploring the chemo-textural familiarity hypothesis for scorpion navigation." The Journal of arachnology 45(3), 265-270. https://doi.org/10.1636/joa-s-16-070.1.

View This Item Online: https://www.biodiversitylibrary.org/item/274724

DOI: https://doi.org/10.1636/joa-s-16-070.1

Permalink: https://www.biodiversitylibrary.org/partpdf/289911

\section{Holding Institution}

Smithsonian Libraries

\section{Sponsored by}

Biodiversity Heritage Library

\section{Copyright \& Reuse}

Copyright Status: In Copyright. Digitized with the permission of the rights holder

Rights Holder: American Arachnological Society

License: https://creativecommons.org/licenses/by-nc-sa/4.0/

Rights: http://www.biodiversitylibrary.org/permissions/

This document was created from content at the Biodiversity Heritage Library, the world's largest open access digital library for biodiversity literature and archives. Visit BHL at https://www.biodiversitylibrary.org. 